\title{
Global tractography of multi-shell diffusion-weighted imaging data using a multi-tissue model
}

\author{
Daan Christiaens $^{\mathrm{a}, \mathrm{g}, *}$, Marco Reisert ${ }^{\mathrm{b}}$, Thijs Dhollander ${ }^{\mathrm{a}, \mathrm{c}, \mathrm{g}}$, Stefan Sunaert ${ }^{\mathrm{d}, \mathrm{e}, \mathrm{g}}$, Paul Suetens ${ }^{\mathrm{a}, \mathrm{f}, \mathrm{g}}$, Frederik Maes ${ }^{\mathrm{a}, \mathrm{g}}$ \\ ${ }^{a} K U$ Leuven, Department of Electrical Engineering, ESAT/PSI, Medical Image Computing, Leuven, Belgium \\ ${ }^{b}$ University of Freiburg Medical Center, Department of Radiology, Medical Physics, Freiburg, Germany \\ ${ }^{c}$ Florey Institute of Neuroscience and Mental Health, Melbourne, Victoria, Australia \\ ${ }^{d} K U$ Leuven, Department of Imaging $\&$ Pathology, Translational MRI, Leuven, Belgium \\ ${ }^{e}$ UZ Leuven, Department of Radiology, Leuven, Belgium \\ ${ }_{i}$ Minds, Medical IT Department, Leuven, Belgium \\ ${ }^{g}$ UZ Leuven, Medical Imaging Research Center, Herestraat 49 box 7003, 3000 Leuven, Belgium
}

\begin{abstract}
Diffusion-weighted imaging and tractography provide a unique, non-invasive technique to study the macroscopic structure and connectivity of brain white matter in vivo. Global tractography methods aim at reconstructing the full-brain fibre configuration that best explains the measured data, based on a generative signal model. In this work, we incorporate a multi-shell multi-tissue model based on spherical convolution, into a global tractography framework, which allows to deal with partial volume effects. The required tissue response functions can be estimated from and hence calibrated to the data. The resulting track reconstruction is quantitatively related to the apparent fibre density in the data. In addition, the fibre orientation distribution for white matter and the volume fractions of grey matter and cerebrospinal fluid are produced as ancillary results. Validation results on simulated data demonstrate that this data-driven approach improves over state-of-the-art streamline and global tracking methods, particularly in the valid connection rate. Results in human brain data correspond to known white matter anatomy and show improved modelling of partial voluming. This work is an important step towards detecting and quantifying white matter changes and connectivity in healthy subjects and patients.
\end{abstract}

Keywords: diffusion-weighted imaging, global tractography, multi-shell multi-tissue model

\section{Introduction}

Diffusion-weighted imaging (DWI) (Le Bihan et al., 1986) and tractography (Mori and van Zijl, 2002) provide a unique, non-invasive technique to study the macroscopic structure and connectivity of the white matter in the human brain in vivo (the human connectome) (Tournier et al., 2011; Dell'Acqua and Catani, 2012). Not only is mapping the connectome one of the biggest challenges in modern neuroscience, a detailed understanding of its structure and organization may also help the neuroscientific community to gain insight in a number of important disease processes (Sporns et al., 2005; Jbabdi and Johansen-Berg, 2011). Therefore, diffusion-weighted imaging and tractography are key elements in recent, large-scale efforts for mapping the human brain (Van Essen et al., 2013; Assaf et al., 2013). Yet, besides large datasets, improved analysis pipelines are needed before connectomics may reliably answer those questions, first and foremost improved microstructural modelling and tractography (Jbabdi and Johansen-Berg, 2011).

While it has been recognized early on that diffusion is sensitive to the underlying fibre geometry (Beaulieu, 2002),

\footnotetext{
* Corresponding author

Email address: daan.christiaens@esat.kuleuven.be (Daan Christiaens)
}

understanding the precise link between both is essential for accurate and robust interpretation of the measured data (Jbabdi and Johansen-Berg, 2011; Mangin et al., 2013). Hence, considerable effort has gone to modelling this socalled local inverse problem, beyond the (Gaussian) diffusion tensor model (Basser et al., 1994). On the one hand, a growing class of methods aims at modelling the biophysical process directly, hence deriving microstructural properties such as axon diameter, neurite density, etc. (Panagiotaki et al., 2012). On the other hand, data-driven approaches have been developed, which aim at deriving the fibre geometry with as little prior assumptions about its physical properties as possible. Arguably the most popular among these are spherical deconvolution (SD) techniques (Tournier et al., 2007; Descoteaux et al., 2009), which reconstruct the fibre orientation distribution function (fODF) based on a fibre response function that may be estimated from the data itself. However, despite the progress in this area, the local inverse problem is inherently incomplete, as the symmetric nature of the diffusion profile cannot discriminate crossing and fanning fibre geometries on a larger scale (Jbabdi and Johansen-Berg, 2011).

Because of the aforementioned limitations of local modelling, Mangin et al. (2013) recently advocated "a shift toward a global inverse problem perspective, namely the global reconstruction of the geometry of the complete white 
matter". Indeed, accounting for the spatial continuity of neural fibres may help in recovering locally ambiguous configurations, and improve the robustness of the model fitting. Such is the motivation behind a growing class of spatially regularized fODF reconstruction methods (Goh et al., 2009; Reisert and Kiselev, 2011; Zhou et al., 2014). Global tractography (GT) methods (Poupon et al., 2000; Mangin et al., 2002; Kreher et al., 2008; Fillard et al., 2009; Reisert et al., 2011, 2014) go even further and aim at reconstructing the entire fibre configuration that best explains the measured diffusion data. Moreover, they address the ill-posed nature of diffusion tractography at the same time, i.e., they are more robust to noise and local reconstruction errors than streamline tracking (Mangin et al., 2013).

Yet, current GT methods rely on specific microstructural models with fixed parameters, which may not always be adapted to the type of data available. Kreher et al. (2008) and Fillard et al. (2009) model the fibre response as an axially-symmetric diffusion tensor. Reisert et al. (2011) use the stick model for the intra-axonal compartment (Behrens et al., 2003), which they have recently extended with a separate extra-axonal compartment, modelled by a diffusion tensor (Reisert et al., 2014). Besides having to be tuned to the data at hand, these models are typically defined for white matter and therefore fail to take partial volume effects from adjacent tissues into account.

In this paper, we introduce a multi-shell spherical harmonic response function, measured from the data, into the generative model defined as part of the global tractography method of Reisert et al. (2011). In addition, we adopt the multi-tissue model of Jeurissen et al. (2014) to differentiate between white matter (WM), grey matter (GM) and cerebrospinal fluid (CSF) compartments. As such, our approach explicitly accounts for partial volume effects, and does not require a white matter mask in the reconstruction.

\section{Methods}

\subsection{Global tractography in the spherical harmonics basis}

Particle-based global tractography methods typically model the neural fibre trajectories as chains of particles (line segments), each characterised by their position $\vec{x}_{i}$ and orientation $\vec{n}_{i}$ (Kreher et al., 2008; Fillard et al., 2009; Reisert et al., 2011). The fibre model $\mathcal{M}$ then consists of the set of all segments $\left\{X_{i}=\left(\vec{x}_{i}, \vec{n}_{i}\right)\right\}$ and a set of connections between their endpoints. Ultimately, we wish to maximize the posterior probability of $\mathcal{M}$ given the data $D$, which, according to Bayes' rule and assuming a Gibbs distribution at temperature $T$, can be written as

$$
\begin{aligned}
P(\mathcal{M} \mid D) & \propto P(D \mid \mathcal{M}) P(\mathcal{M}) \\
& =e^{-E_{\text {data }}(\mathcal{M}, D) / T} e^{-E_{\mathrm{con}}(\mathcal{M}) / T} .
\end{aligned}
$$

As such, the problem becomes finding the global minimum of $E(\mathcal{M})=E_{\text {data }}(\mathcal{M}, D)+E_{\text {con }}(\mathcal{M})$. The data energy $E_{\text {data }}$ relates to the data likelihood and is defined as the mean squared error between the measured data $D$ and the predicted data $D^{\prime}$, simulated from the particle configuration $\mathcal{M}$ using a generative model. The connection energy $E_{\text {con }}$ relates to the model prior and promotes connectivity and smoothness of the reconstructed tracks.

\subsubsection{Generative model}

The central hypothesis in this work is that, for white matter, each segment has a fixed and equal contribution to the predicted data $D_{\mathrm{WM}}^{\prime}$, in the form of a fibre response kernel $K_{b}(\theta) . \quad K_{b}$ is a spherical function depending only on the elevation angle $\theta$ and the $b$-value, that models the expected diffusion signal for a single fibre direction along the $z$-axis. As such, we can simulate the white matter signal for gradient direction $(\vec{g}, b)$ by orienting the $z$-axis of this kernel along all segments, and integrating over all segments in a voxel $\vec{r}$, i.e.,

$$
D_{\mathrm{WM}}^{\prime}(\vec{r}, \vec{g}, b)=\sum_{\substack{\left(\vec{x}_{i}, \vec{n}_{i}\right) \\ \vec{x}_{i} \in \mathcal{N}(\vec{r})}} w\left(\left\|\vec{r}-\vec{x}_{i}\right\|\right) K_{b}\left(\arccos \left(\vec{n}_{i} \cdot \vec{g}\right)\right)
$$

In this equation, $\mathcal{N}(\vec{r})$ denotes the voxel neighbourhood and $w(\cdot)$ is some spatial weighting function. In the most simple case, $w$ is a block function the size of one voxel. Cast into the basis of real, symmetric spherical harmonics (SH) (Descoteaux et al., 2009), the kernel reorientation can be described as a convolution with a SH Dirac delta function $\delta_{\vec{n}_{i}}$ along the segment direction $\vec{n}_{i}$. As such, the predicted white matter signal becomes

$$
\begin{aligned}
D_{\mathrm{WM}}^{\prime}(\vec{r}, \vec{g}, b)= & \sum_{\substack{\left(\vec{x}_{i}, \vec{n}_{i}\right) \\
\vec{x}_{i} \in \mathcal{N}(\vec{r})}} w\left(\left\|\vec{r}-\vec{x}_{i}\right\|\right)\left(K_{b} * \delta_{\vec{n}_{i}}\right)(\vec{g}) \\
= & K_{b} * \sum_{\substack{\left(\vec{x}_{i}, \vec{n}_{i}\right) \\
\vec{x}_{i} \in \mathcal{N}(\vec{r})}} w\left(\left\|\vec{r}-\vec{x}_{i}\right\|\right) \delta_{\vec{n}_{i}}(\vec{g}) \\
= & K_{b} * \Psi(\vec{r}, \vec{g}),
\end{aligned}
$$

where $*$ is the spherical convolution operator and $\Psi(\vec{r}, \vec{u})$ is an $\mathrm{SH}$ orientation distribution function (ODF) of the segments in voxel $\vec{r}$. Hence, the white matter signal is simulated by converting the segment configuration to a fibre ODF and calculating the convolution with a kernel $K_{b}$, as depicted in Fig. 1.

In addition, similar to Jeurissen et al. (2014), we introduce one or more isotropic kernels $c_{j}(b)$ that account for partial volume contamination of other tissue types. Typically, we will use these to model cerebrospinal fluid (CSF) and grey matter (GM), but it should be noted that these can be used to model any isotropic signal component. Hence our complete model becomes

$$
D^{\prime}(\vec{r}, \vec{g}, b)=K_{b} * \Psi(\vec{r}, \vec{g})+\sum_{j} c_{j}(b) f_{j}(\vec{r}),
$$

where $f_{j}(\vec{r})$ is the fraction of isotropic component $j$ in voxel $\vec{r}$. 
a

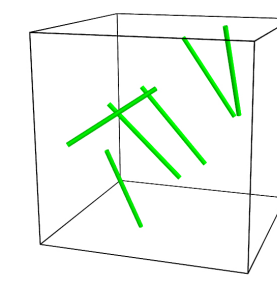

b

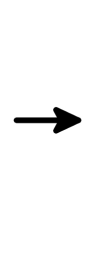

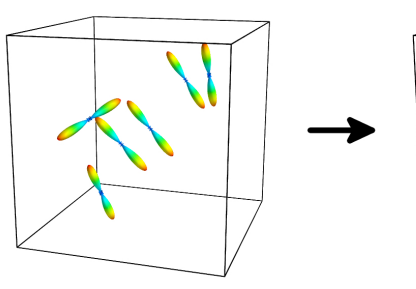

C

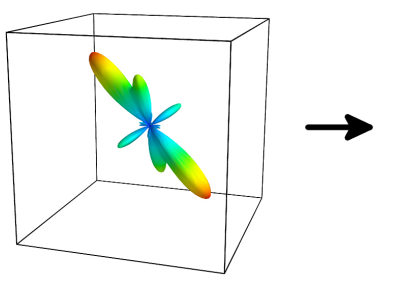

d

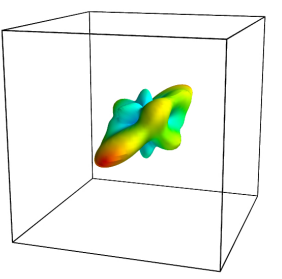

Figure 1: Generative model: (a) consider all particles positioned within a voxel; (b) each of them is represented as a Dirac delta function in the spherical harmonics basis; (c) the sum of these functions is the segment orientation distribution function (ODF); (d) the convolution of this ODF with the fibre response function is the simulated signal $D_{\mathrm{WM}}^{\prime}$.

\subsubsection{Data likelihood and priors}

Assuming a Gaussian data likelihood, the data energy is defined as

$$
E_{\text {data }}(\mathcal{M}, D)=\kappa\left(\frac{\left\|D-D^{\prime}\right\|^{2}}{Q K_{0}^{2}}+\mu N_{p}\right),
$$

in which $\kappa$ is a weighting factor. In the first term, $Q$ is the number of acquired DWI volumes and $K_{0}$ is the amplitude of the $b=0 \mathrm{WM}$ response function. Hence, this term expresses the mean-squared-error of the data relative to the kernel. Because $K_{0}$ is proportional to the intensity of the DWI data, this scaling assures that the reconstruction can handle different acquisition protocols and gradient schemes without needing to adapt the parameters. The second term imposes a $L_{1}$-prior on the total number of particles $N_{p}$ in the model, each of which has an associated cost $\mu$ (the particle potential). As such, we aim to reconstruct the data as good as possible, with as little particles as needed.

The connection energy is defined as in Reisert et al. (2011),

$$
E_{\text {con }}(\mathcal{M})=\lambda\left(\sum_{X_{i}-X_{j} \in \mathcal{M}} U_{\text {bend }}\left(X_{i}-X_{j}\right)-\nu N_{c}\right) .
$$

The first term comprises the total bending energy across all connected segment pairs $X_{i}-X_{j}$, expressed as the distance of the connected endpoints to the midpoint between both segments. The second term introduces a connection potential $\nu$ for each connection, with $N_{c}$ the total number of connections, meant to encourage particles to connect to their neighbouring segments.

The weighting factors $\kappa$ and $\lambda$ of the data and connection energy terms determine the balance between both. Generally, a good balance depends on the required number of particles, which is controlled by the segment weight $w$ as defined in (3). Additionally, we gradually decrease the weight of the data energy in favour of connection energy throughout the optimization, controlled by the temperature $T$ (see below). In practice, $\kappa=\frac{T}{T_{1}} \frac{1}{w^{2}}$ and $\lambda=1$ is a good default setting for different datasets. The remaining two parameters, the particle and connection potentials, can be adapted by the user to give more or less weight to the priors.

\subsubsection{Optimization}

The optimization of (1) is achieved with a Reversible jump Markov Chain Monte Carlo (RJMCMC) algorithm (Green, 1995), which allows to obtain random samples from the posterior distribution $P(\mathcal{M} \mid D)$. As in simulated annealing methods, the temperature $T$ is gradually cooled down to increase the likelihood of sampling from the maximum of $P(\mathcal{M} \mid D)$ (or the minimum of $E(\mathcal{M})$ ). At each iteration, the algorithm proposes a new state $\mathcal{M}^{\prime}$, obtained as a random perturbation of the current state $\mathcal{M}$, and evaluates the Green's ratio

$$
R=\min \left(1, \frac{e^{-E\left(\mathcal{M}^{\prime}\right) / T}}{e^{-E(\mathcal{M}) / T}} \frac{p^{\operatorname{prop}}\left(\mathcal{M} \mid \mathcal{M}^{\prime}\right)}{p^{\operatorname{prop}}\left(\mathcal{M}^{\prime} \mid \mathcal{M}\right)}\right),
$$

where $p^{\text {prop }}$ (next|current) is the transition probability from current state to the next state. The proposed state $\mathcal{M}^{\prime}$ is then accepted with probability $R$, or discarded otherwise. Transition proposals include creating or removing a particle, changing its position and orientation by either a random perturbation or optimally w.r.t.its connected neighbours, and (dis)connecting a particle's endpoint to the endpoint of another particle in its neighbourhood (Kreher et al., 2008; Reisert et al., 2011).

Upon every change to the particles in voxel $\vec{r}$, the isotropic fractions $f_{j}(\vec{r})$ are updated by evaluating the difference between the measured data $D$ and the predicted WM signal $D_{\mathrm{WM}}^{\prime}$. By definition (7), this difference equals the other tissue compartments, as well as the residual WM, not yet recovered by the optimization. To account for the latter, we introduce an additional isotropic fraction of residual WM $f_{\text {resWM }}$ with corresponding kernel $c_{\mathrm{WM}}(b)$, defined as the isotropic part of the white matter kernel $K_{b}(\theta)$. All isotropic fractions, including the residual WM, are then estimated as a non-negativity constrained, linear least-squares fit to

$$
D-D_{\mathrm{WM}}^{\prime}=\sum_{j} c_{j}(b) f_{j}+c_{\mathrm{WM}}(b) f_{\mathrm{resWM}} .
$$

Finally, the data energy is updated by evaluating (7)-(8).

The presented method was implemented as an add-on to MRtrix (J-D Tournier, Brain Research Institute, Mel- 
bourne, Australia, https://github.com/MRtrix3/mrtrix3), which we plan to release to the community.

\subsection{Response function estimation}

So far, we have made no prior assumptions on the acquisition protocol. The presented method can be applied to data of any $\vec{q}$-space sampling scheme, as long as $K_{b}(\theta)$ can be defined. For the remainder of this paper, we choose to estimate the fibre response function from the data, which requires a multi-shell protocol. The main advantage of this approach is that $K_{b}$ is easily calibrated to the scanner, the acquisition sequence, and the (group of) subject(s). The white matter response function is estimated as the average signal in a mask of single fibre voxels, after reorienting the gradient tables in these voxels such that the principal eigenvectors of the diffusion tensors align (Tournier et al., 2007). CSF and GM kernels are estimated as the mean DWI signal per shell, averaged across respective masks.

A single fibre WM mask and isotropic GM and CSF masks can be obtained from a T1w-image and the fractional anisotropy (FA) map, provided that the T1w and DWI data have been co-registered to the same space (see Data below). First, a probabilistic tissue segmentation is generated from a T1w-image using FSL FAST (Zhang et al., 2001) and thresholded at probability $>95 \%$ for WM, GM and CSF separately. Secondly, single fibre voxels are selected from the WM segmentation by thresholding the FA above 0.75 . Similarly, isotropic GM and CSF voxels are selected by imposing an FA below 0.1. An example of the resulting masks and kernels are shown in Fig. 2.

\section{Data and Experiments}

\subsection{Data}

\subsubsection{Simulated phantom}

Phantom data with known ground truth was generated using the Phantomas software (Caruyer et al., 2014), based on the geometry of the 2nd HARDI reconstruction challenge, organized at ISBI 2013 (Daducci et al., 2013). This phantom consists of white matter, grey matter, and CSF mimicking regions, simulated with realistic relaxation times. The white matter contains 27 fibre bundles, including crossing, kissing, and fanning structures, simulated with a multi-tensor model with principal eigenvalue 0.0017 and secondary eigenvalues 0.0002 , and corrupted with Rician noise at signal-to-noise ratios (SNR) 5, 10, 20, 30, 40, and 50. The voxel size is $2 \mathrm{~mm}$ isotropic; the gradient encoding scheme equals that of the Human Connectome Project (cf. in vivo dataset 1). All tissue-specific response functions are estimated in ground-truth regions on the noise-free dataset.

\subsubsection{In vivo dataset 1}

Data of a neurologically healthy subject was provided by the WU-Minn Human Connectome Project (HCP) Q3 data release (Van Essen et al., 2013). The diffusion data consists of $3 \times 90$ gradient directions at $b$-values 1000 , 2000 , and $3000 \mathrm{~s} / \mathrm{mm}^{2}$ and 18 non-diffusion weighted images $(b=0)$, at an isotropic voxel size of $1.25 \mathrm{~mm}$, and was corrected for motion, eddy current, and EPI distortions as described in Glasser et al. (2013). In addition, a T1weighted image of isotropic voxel size $0.7 \mathrm{~mm}$ is available in the same reference frame.

\subsubsection{In vivo dataset 2}

A second, healthy subject was scanned on a Philips Achieva 3.0 T TX system, using a diffusion-weighted imaging sequence comprised of $10 b=0$ images, 25 images at $b$-value $700 \mathrm{~s} / \mathrm{mm}^{2}, 40$ images at $b=1000 \mathrm{~s} / \mathrm{mm}^{2}$, and 75 images at $b=2800 \mathrm{~s} / \mathrm{mm}^{2}$. The voxel size equals $2.5 \mathrm{~mm}$ (isotropic). Additionally, a single $b=0$ image was acquired with reverse-phase encoding to correct for EPI distortions, as well as motion and eddy current distortions using FSL TOPUP and EDDY tools (Andersson et al., 2003; Smith et al., 2004). A T1w-scan was acquired with a 3D TFE sequence, at voxel size $1.2 \mathrm{~mm} \times 0.98 \mathrm{~mm} \times 0.98 \mathrm{~mm}$.

\subsection{Parameter settings}

The maximal $\mathrm{SH}$ order $\ell_{\max }=10$ was used in all experiments. In the phantom dataset, we use a segment length $2 \ell=4 \mathrm{~mm}$ and a particle weight $w=0.1$, meaning that on average $w^{-1}=10$ segments per voxel are needed to reconstruct the white matter. For the in vivo datasets, the segment length $2 \ell$ is $2 \mathrm{~mm}$. The particle weight $w=0.1$ for dataset 1 and $w=0.0125$ for dataset 2 , to account for the larger voxel size. The connection potential $\nu$ is fixed at 0.5 for all experiments. The particle potential is set to $\mu=5 \% w$.

The optimization starts with a burn-in phase at constant temperature $T_{0}$ for the first $10 \%$ of the iterations, and then proceeds with an exponential cooling schedule towards the final temperature $T_{1}$. For the phantom dataset, we used $10^{8}$ iterations from $T_{0}=0.5$ to $T_{1}=0.001$. For the in vivo data, we used $10^{9}$ iterations, with $T_{0}=0.1$ and $T_{1}=0.001$.

\subsection{Validation}

We compare the proposed multi-shell multi-tissue global tractography (MSMT-GT) method to two alternative (multishell) approaches on the simulated phantom:

1. Multi-shell multi-tissue constrained spherical deconvolution (MSMT-CSD) (Jeurissen et al., 2014), followed by probabilistic streamline tractography using the 2nd order Runge-Kutta method iFOD2 as implemented in MRtrix (Tournier et al., 2010, 2012). All parameters are set to their default values: the step size is $0.5 \times$ voxel size, the maximum angle between consecutive steps is $45^{\circ}$, the fODF amplitude cut-off is 0.1 , and the minimum track length is $5 \times$ voxel size. Seed points are drawn from a uniform distribution in a full brain mask. 

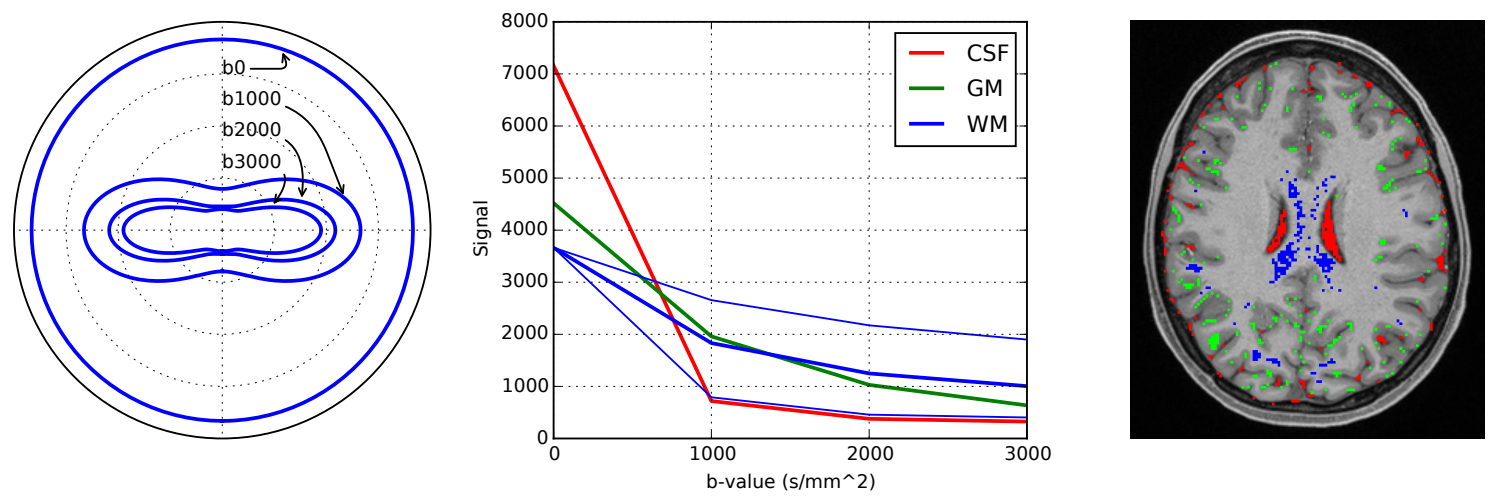

Figure 2: Estimated kernels for dataset 1. Left: the fibre response kernel for a fibre along the vertical axis, at different $b$-values. Middle: the radial attenuation of the CSF and GM kernels, as well as the isotropic part of the WM kernel (thick blue line) and its radial (top thin line) and axial attenuation (bottom thin line). Right: Corresponding masks in which these kernels are estimated.

2. The global tractography method of Reisert et al. (2011), as implemented in MITK (Neher et al., 2012; Fritzsche et al., 2012), which evaluates the ball-andstick model on the diffusion ODF (Tuch et al., 2002). We employed the multi-shell diffusion ODF reconstruction method of Aganj et al. (2010), and set all common parameters such as segment length and temperature equal to those chosen in our own method. Parameters specific to the ball-and-stick model were tuned by MITK with the built-in methods within a WM mask.

Quantitative comparison in the simulated datasets is based on the tractometer approach (Côté et al., 2013; Houde et al., 2014; Girard et al., 2014). Given regions-of-interest (ROIs) at the ends of all bundles in the phantom, we calculate the percentage of valid connections (VC) and invalid connections (IC) between these end regions, and the percentage of no connections (NC), i.e., tracks that reach none or only one of the target ROIs. Ideally, $\mathrm{VC}$ is $100 \%$ and $\mathrm{IC}$ and $\mathrm{NC}$ are $0 \%$. For ease of interpretation, we also report the total connection ratio $\mathrm{VC}+\mathrm{IC}=1-\mathrm{NC}$, which should be large for maximal connectivity, and the valid connection ratio $\mathrm{VC} /(\mathrm{VC}+\mathrm{IC})$, which should be large for maximal specificity. Finally, we count the number of valid bundles (VB) and invalid bundles (IB), where a bundle is counted as soon as at least one track connects its two end regions. The phantom contains 27 ground truth bundles between 53 end regions, which allows for a maximum of 1351 invalid bundles.

In the in vivo datasets, we provide a visual comparison between these methods in interesting regions and fibre bundles, which allows for a qualitative assessment of the reconstructed tractograms.

\section{Results}

\subsection{Simulated data}

We investigate the effect of the particle potential $\mu$ and connection potential $\nu$ on the tractometer analysis. First, fixing $\nu=0.5$, the effect of varying $\mu$ is shown on the left in Fig. 3. The total connectivity $\mathrm{VC}+\mathrm{IC}$ is maximum at $\mu=2 \% w(23.9 \%)$, and the valid connection ratio $\mathrm{VC} /(\mathrm{VC}+\mathrm{IC})$ reaches a maximum of $69.9 \%$ for $\mu=$ $1 \% \mathrm{w}$. The number of valid bundles $\mathrm{VB}$ is 27 for most of the range but decreases at $\mu=10 \% w$ and beyond. The number of invalid bundles IB shows a clear decreasing trend for increasing particle potential. Secondly, when fixing $\mu=5 \% \mathrm{w}$ and varying the connection potential $\nu$, the total connection ratio increases for higher $\nu$, while the valid connection ratio decreases. Simultaneously, both VB and IB increase for increasing $\nu$. As such, decreasing the connection potential improves the precision while sacrificing connectivity. It is clear from these graphs that there is no single parameter setting that performs best by all measures. We therefore selected $\nu=0.5$ and $\mu=5 \% w$ as a sensible compromise.

Given these parameters, we compare the presented method to the competing techniques described above across different SNR levels. The resulting metrics are plotted in Fig. 4. For SNR 20, we listed the results in Table 1 and depicted the reconstructed tracks in Fig. 5. The presented MSMT-GT method has the best valid connection ratio of the tested techniques, especially for $\mathrm{SNR} \geq 10$. Streamline tractography consistently outperforms global tractography in terms of the total connection rate. The multi-shell multitissue model proves particularly beneficial in the low-SNR range $(\leq 20)$ for both streamline and global tractography, as shown by the higher $\mathrm{VC} /(\mathrm{VC}+\mathrm{IC})$ and much lower IB. This can also be observed in Fig. 5, which shows that MITK-GT results in many spurious tracks in GM regions that are not explicitly accounted for in its ball-and-stick model. Additionally, MITK was never able to reconstruct 

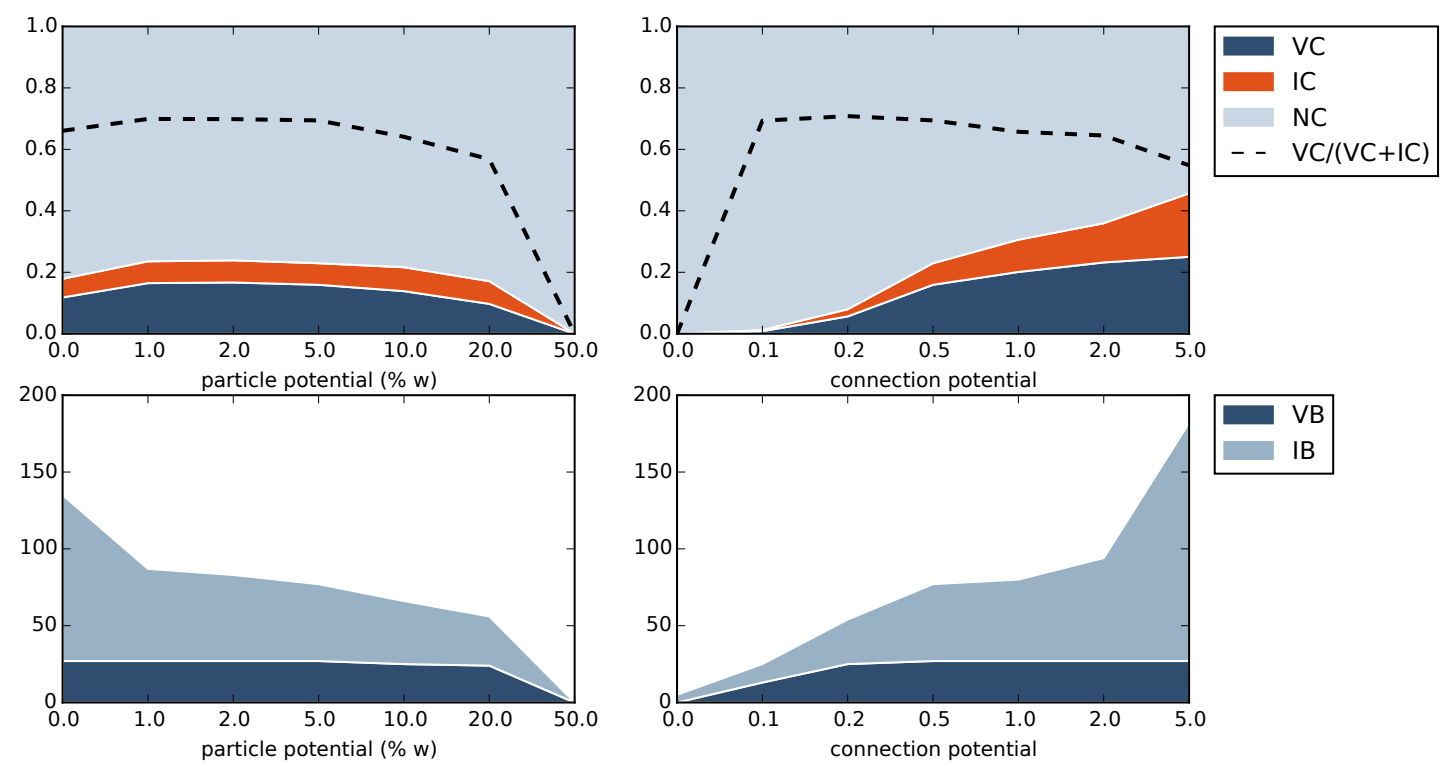

Figure 3: Tractometer analysis of the simulated phantom: effect of the particle and connection potentials at SNR 20. In the left plots, the particle potential $\mu$ (expressed as a percentage of the particle weight $w$ ) is adapted while the connection potential is kept constant at $\nu=0.5$. In the plots on the right, $\nu$ is adapted while $\mu$ is fixed at $5 \% \mathrm{w}$.
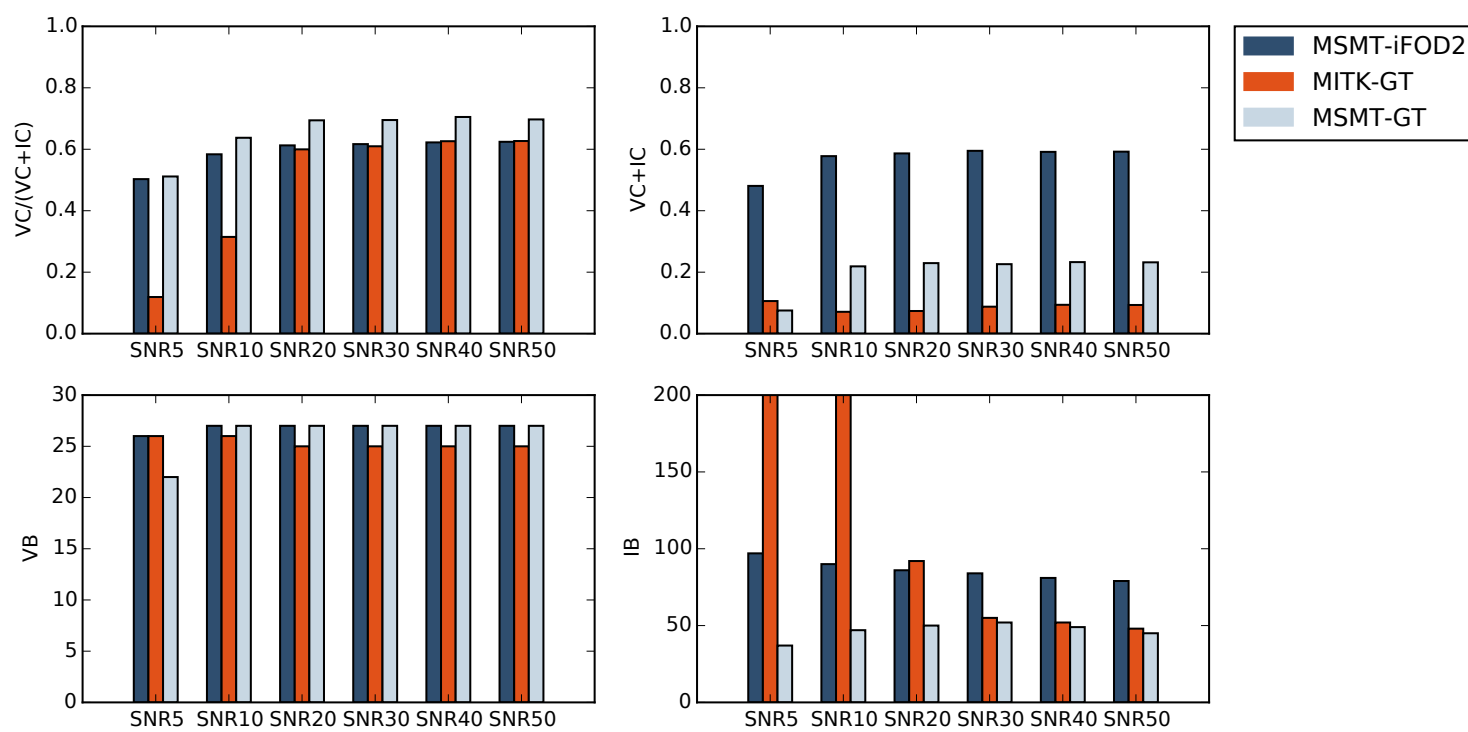

Figure 4: Tractometer analysis of the simulated phantom: comparison to streamline tractography and MITK global tractography on for different SNR levels. 
Table 1: Tractometer results of the simulated phantom at SNR 20. The number of valid connections (VC), invalid connections (IC), and no connections (NC) are expressed as percentages relative to the total number of tracks (\#t). The numbers of valid and invalid bundles (VB and IB) are expressed in absolute terms.

\begin{tabular}{lrrrccccc}
\hline & \#t & $\mathrm{VC}(\%)$ & $\mathrm{IC}(\%)$ & $\mathrm{NC}(\%)$ & $\mathrm{VC}+\mathrm{IC}(\%)$ & $\frac{\mathrm{VC}}{\mathrm{VC}+\mathrm{IC}}(\%)$ & $\mathrm{VB}$ & $\mathrm{IB}$ \\
\hline MSMT-iFOD2 & 10000 & 35.9 & 22.7 & 41.4 & $\mathbf{5 8 . 6}$ & 61.3 & $\mathbf{2 7}$ & 86 \\
MITK-GT & 8511 & 4.4 & 3.0 & 92.6 & 7.4 & 59.5 & 25 & 92 \\
MSMT-GT & 5756 & 15.9 & 7.0 & 77.1 & 22.9 & $\mathbf{6 9 . 4}$ & $\mathbf{2 7}$ & $\mathbf{5 0}$ \\
\hline
\end{tabular}

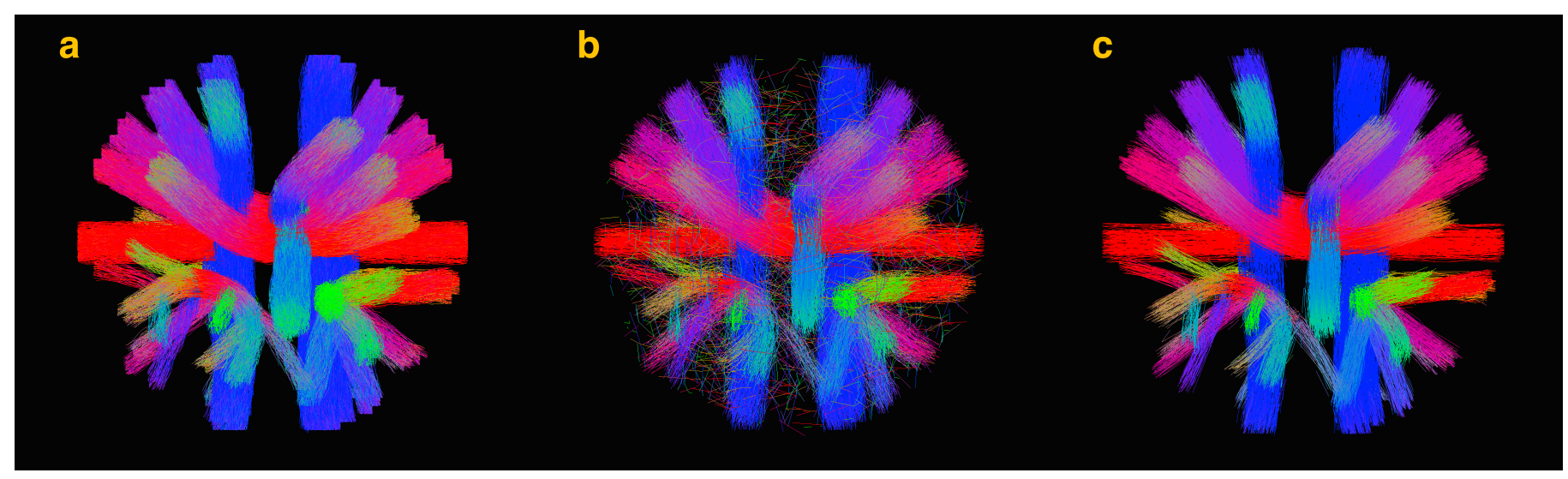

Figure 5: Reconstruction of the simulated phantom at SNR 20: (a) probabilistic streamline tracking on the fODF, reconstructed using multi-shell multi-tissue CSD, (b) MITK global tractography, and (c) our method using the multi-shell multi-tissue model.

all 27 fibre bundles in the phantom, while both MSMT approaches did for sufficient SNR.

\subsection{In vivo data}

The total processing time of MSMT-GT for a full brain tractogram with the specified parameter settings was about $4 \mathrm{~h}$ for dataset 1 and $3 \mathrm{~h}$ for dataset 2 (standard desktop running 4 parallel threads). By comparison, MITK-GT took around $4 \mathrm{~h}$ for dataset 1 and $2 \mathrm{~h}$ for dataset 2 . Streamline tracking (iFOD2) is much faster, but full-brain MSMTCSD still takes over $4 \mathrm{~h}$ in dataset 1 and around $30 \mathrm{~min}$ in dataset 2 .

The estimated volume fractions of all tissues are shown in Fig. 6 for both datasets. The segment density map (WM fraction), and the CSF and GM fractions, have the contrast expected from these tissues, although the segment density exhibits a slight drop in crossing fibre regions. The residual error map is expressed as the root-mean-squared (RMS) error between measured and simulated data, relative to the WM scaling, i.e., the square root of the first term in (8): $\left\|D-D^{\prime}\right\| / \sqrt{Q} K_{0}$. This relative error is fairly homogeneous in each tissue and around $2 \%$ in WM. Ventricular CSF stands out due to its strong attenuation and high signal at $b=0$, whereas WM is affected by the particle potential.

In Figs. 7-9, we show close-ups of the tractography results obtained with the proposed MSMT-GT method for the semioval centre, the fornix, and the precentral gyrus, and compare these visually to the results of MITK global tractography and MSMT-CSD. In Fig. 7 for dataset 2, the tracks from the corpus callosum radiate laterally, crossing the corona radiata and the superior longitudinal fasciculus. This can also be observed in the fODFs, displayed at the bottom of that figure, which are produced as an ancillary result of our global tractography approach and closely resemble the fODFs produced by MSMT-CSD. With MITKGT, we observe spurious fibres at the edge of the ventricles and little tracks radiating laterally. Fig. 8 shows part of the fornix in the mid-sagittal plane, as well as the corpus callosum and the anterior commissure, overlaid on a map of the estimated CSF fraction. The partial volume effect between the ventricles and the surrounding WM structures is explicitly modelled and introduced little to no spurious peaks in the fODFs. Compared to MITK-GT, our approach produced less spurious fibres in PVE regions. Fig. 9 shows the association fibres connecting grey matter areas in the motor cortex (precentral gyrus) and the premotor cortex (middle frontal gyrus). Compared to MSMT-CSD, our approach underestimates the WM fraction at the WM-GM interface due to the imposed particle potential. Without such prior, tracks run deeper into cortical GM, but also falsely connect through the precentral sulcus.

In addition, we segmented white matter bundles based on the protocol outlined in Wassermann et al. (2013), which queries tracks based on their anatomical position w.r.t. a cortical parcellation. We show 5 segmented fibre bundles in Fig. 10 and compare them qualitatively between methods. For the given segmentation, iFOD2 streamline tracking produces dense fibre bundles which successfully delineate 


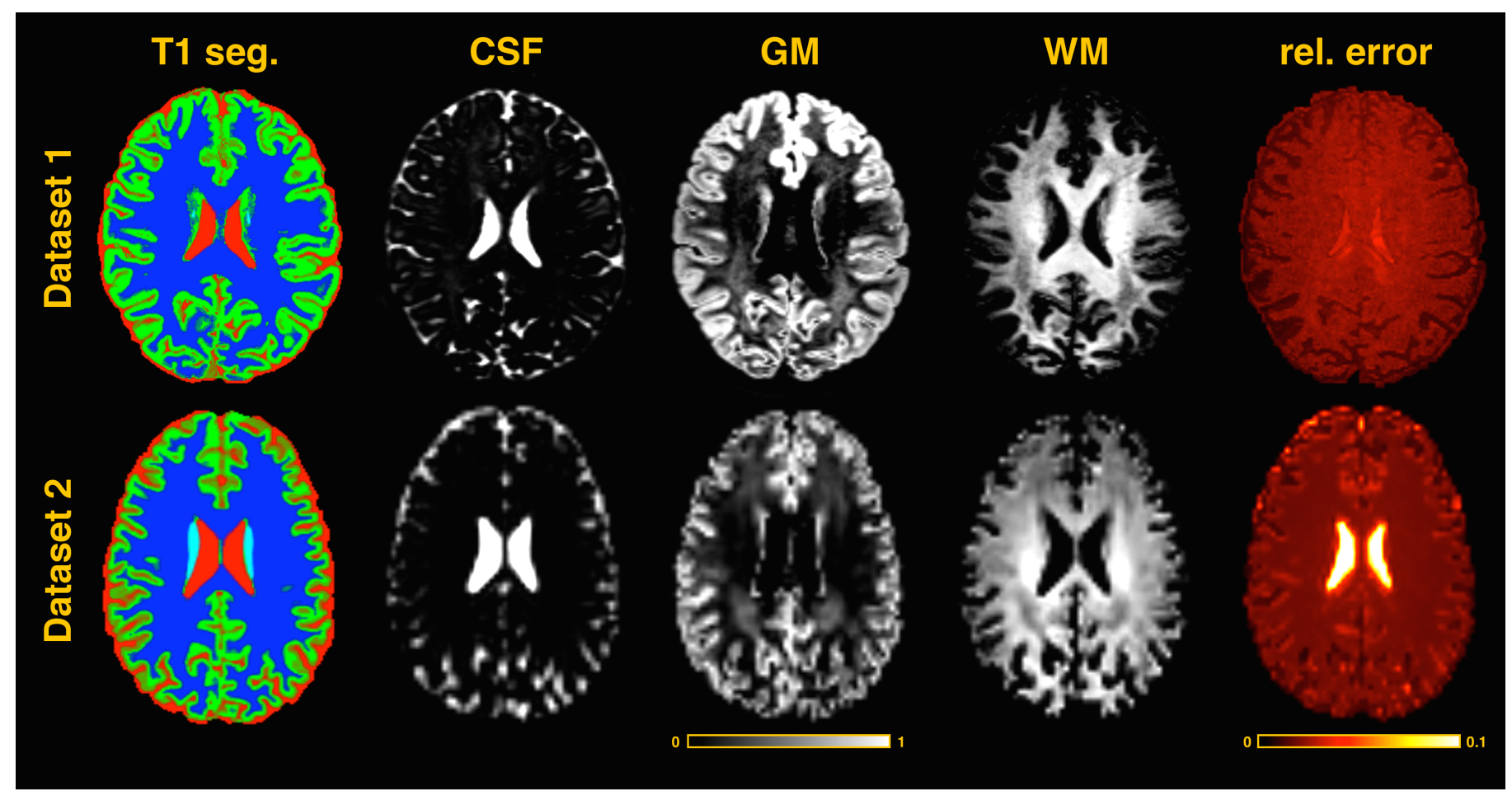

Figure 6: Axial slices of the estimated tissue fractions in both datasets: (a) a T1 segmentation for reference, (b) the fraction of cerebrospinal fluid (CSF), (c) the fraction of grey matter (GM), (d) the fraction of white matter (WM), proportional to the segment density, and (e) the residual error map, measured as the relative root-mean-squared (RMS) difference.

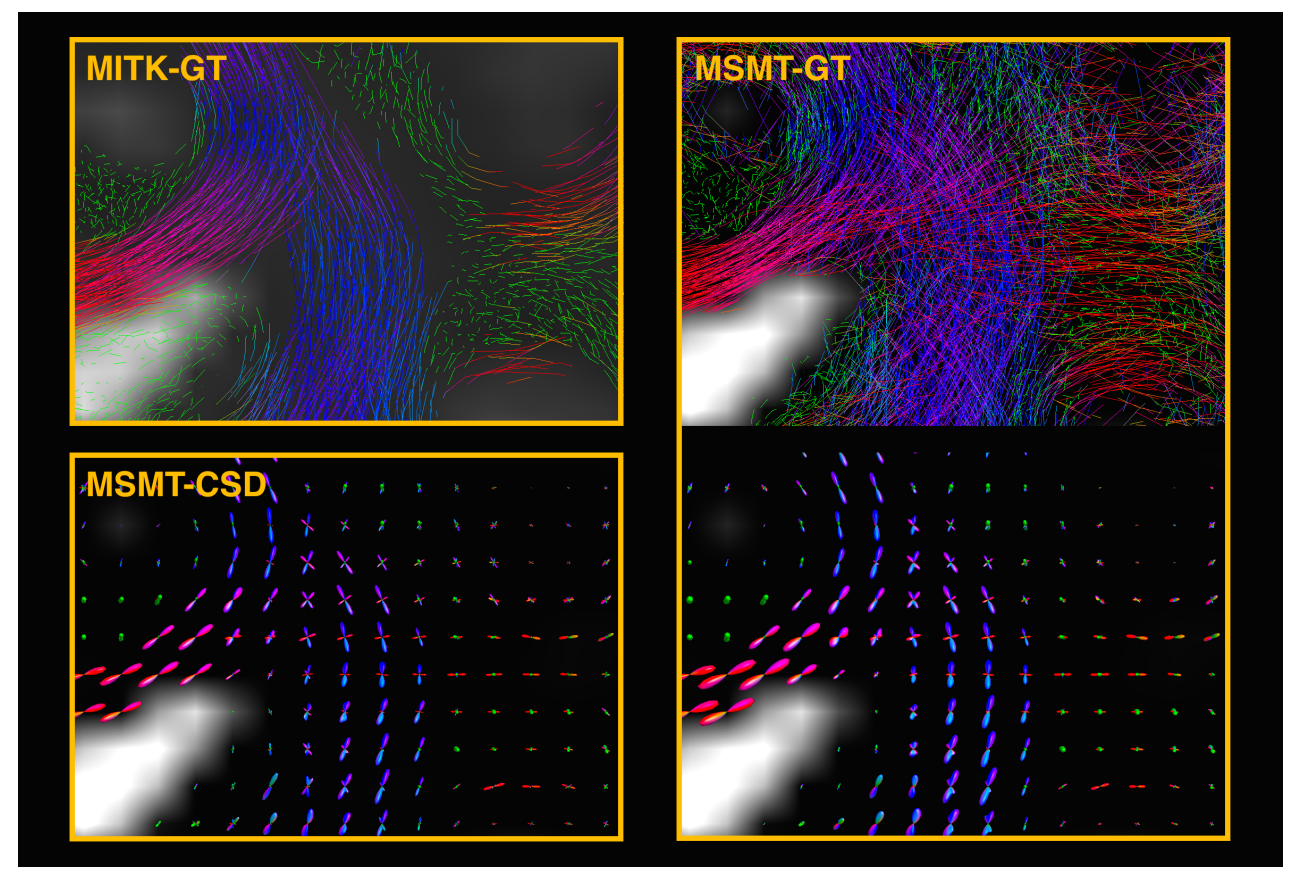

Figure 7: Centrum semiovale of in vivo dataset 2. Right: the tracks within a coronal slab of $2.5 \mathrm{~mm}$ and the associated fODFs, overlaid on the estimated CSF fraction. Top left: corresponding view of the tracks in MITK-GT, overlaid on the $b=0$ image. Bottom left: fODFs in MSMT-CSD, overlaid on the CSF fraction estimated in MSMT-CSD. 

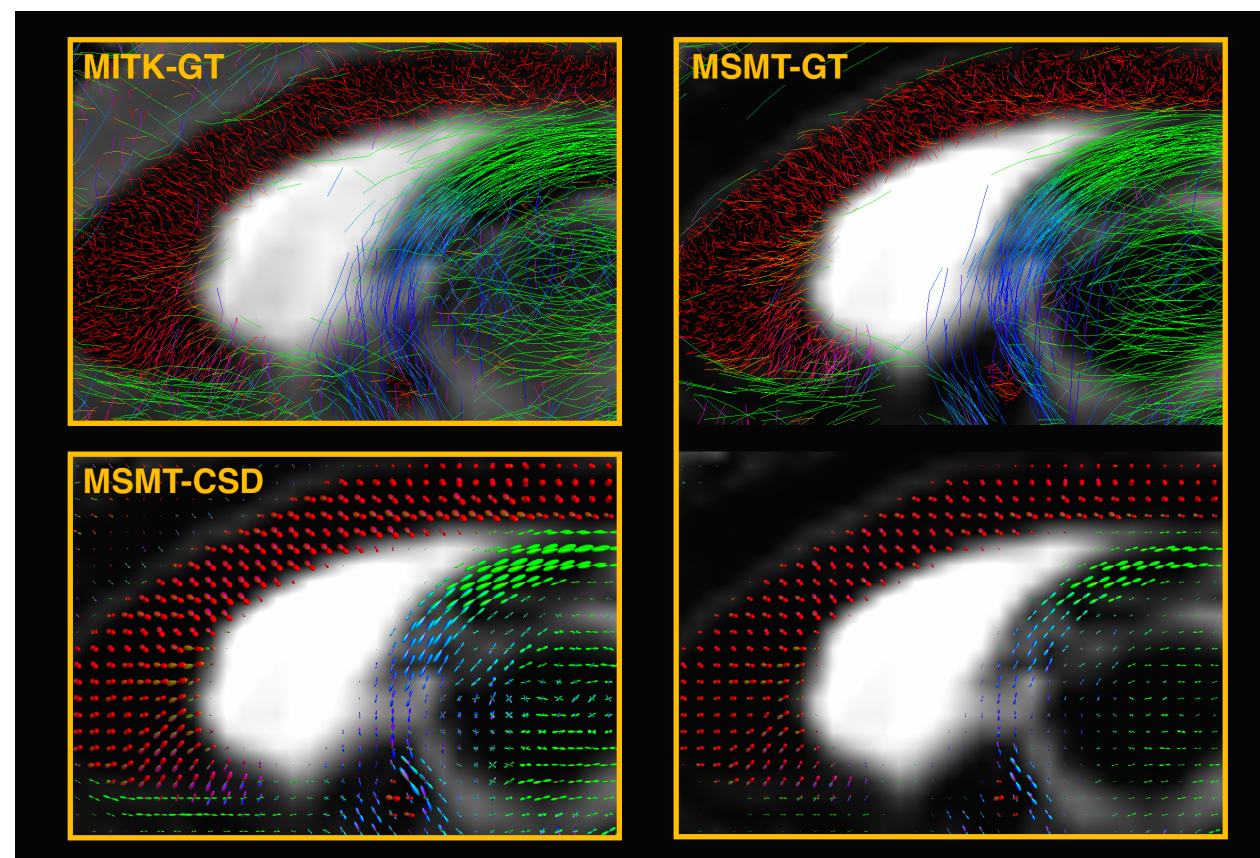

Figure 8: Sagittal view of the fornix of in vivo dataset 1. Right: the tracks within a slab of $2.5 \mathrm{~mm}$ and the associated fODFs, overlaid on the estimated CSF fraction. Top left: corresponding view of the tracks in MITK-GT, overlaid on the $b=0$ image. Bottom left: fODFs in MSMT-CSD, overlaid on the CSF fraction estimated in MSMT-CSD.

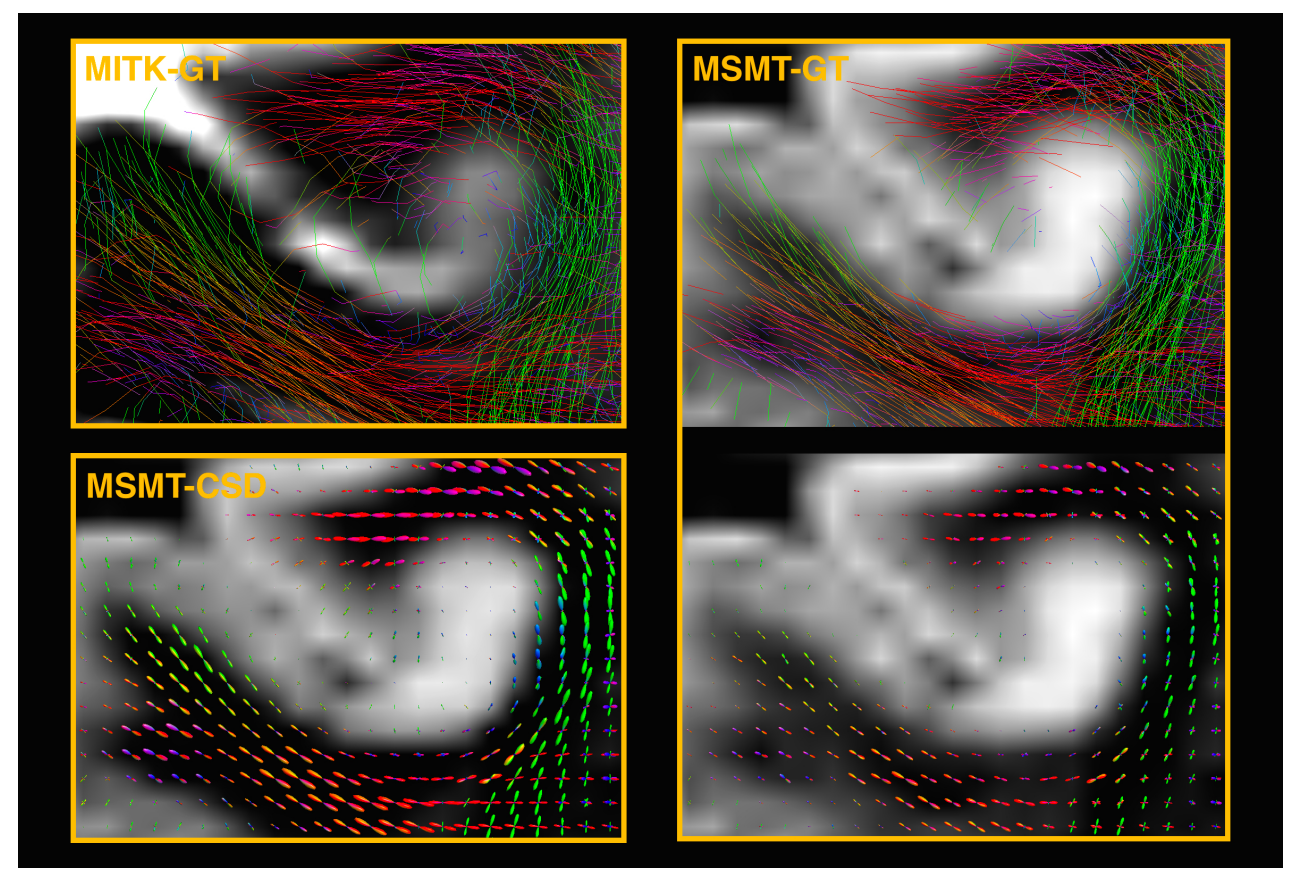

Figure 9: Axial view of the inferior part of the precentral gyrus and the middle frontal gyrus of in vivo dataset 1. Right: the tracks within a slab of $2.5 \mathrm{~mm}$ and the associated fODFs, overlaid on the estimated GM fraction. Top left: corresponding view of the tracks in MITK-GT, overlaid on the $b=0$ image. Bottom left: fODFs in MSMT-CSD, overlaid on the GM fraction estimated in MSMT-CSD. 


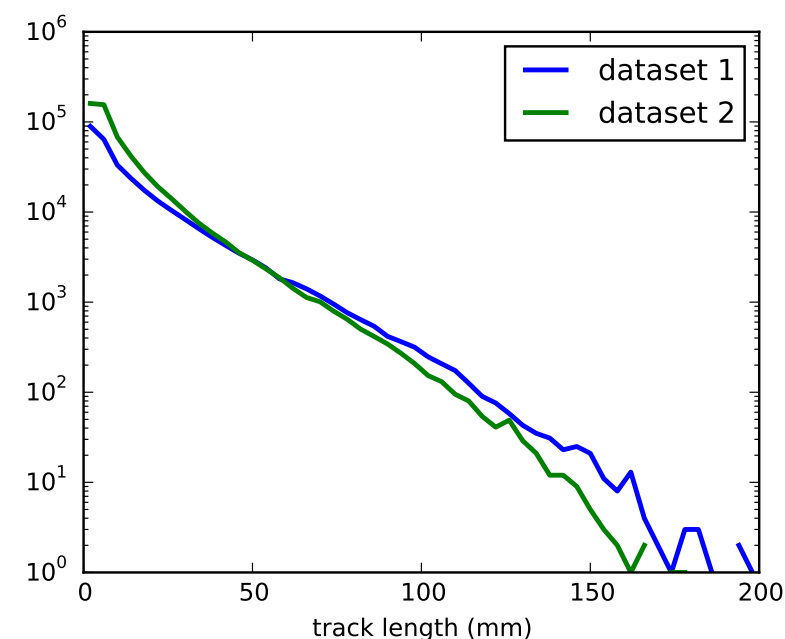

Figure 11: Histogram of the track length, computed across 50 bins in the range of 0 to $200 \mathrm{~mm}$. Notice the log-scale on the vertical axis.

the anatomical structures. Global tractography results are less dense due to many short tracks that do not reach the inclusion ROIs. Nevertheless, a clear improvement of the MSMT model over MITK can be observed, particularly in the corpus callosum and the uncinate fasciculus.

In dataset 1 , we found that $62.9 \%$ of all white matter voxels contains crossing fibres, and $32.2 \%$ contains a 3 way crossing, which is consistent with previous estimates (Jeurissen et al., 2012). The histogram of the track length is shown in Fig. 11 and closely fits an exponential distribution.

\section{Discussion}

\subsection{Multi-shell, multi-tissue global tractography}

Mapping the human connectome in vivo by tractography is only meaningful when the track distribution, if not every track, truly represents some property of the neural tissue. At the very least, the density of the reconstructed full-brain tractogram should correspond to the apparent fibre density (AFD) in the measured DWI data (Raffelt et al., 2012; Dell'Acqua et al., 2013). Streamline tractography, due to its dependence on the seeding distribution and hard thresholds on curvature, FA, or fODF amplitude, lacks any quantitative correspondence to the data and is therefore not suited for measuring connectivity (Jones et al., 2013).

Both generative and discriminative methods have been presented which ensure this correspondence. Discriminative methods (Sherbondy et al., 2010; Smith et al., 2013, 2015; Daducci et al., 2014) start from a dense superset of fibre tracks and filter it until it corresponds to the fODF or the data in a convex optimization framework. Their main advantage is that anatomical constraints can easily be incorporated (Smith et al., 2012; Lemkaddem et al., 2014). On the downside, these methods have been shown to depend on the input superset (Daducci et al., 2014), especially regarding false negatives. On the other hand, generative global tractography methods (Kreher et al., 2008; Fillard et al., 2009; Reisert et al., 2011, 2014) directly optimize the tractogram to the data given a generative signal model and priors on the track configuration.

Our approach extends the generative signal model to arbitrary, multi-shell fibre response functions. While this kernel can be modelled in many ways, we propose to estimate it from the dataset at hand, specific to a subject or study. In this way, the presented approach is not restricted to fixed microstructural models, unlike previous work (Fillard et al., 2009; Reisert et al., 2011). Compared to Reisert et al. (2014), which jointly estimates the global track configuration and the local parameters of a biexponential microstructure model, our approach is less computationally demanding.

In line with Jeurissen et al. (2014), we also extended the generative model for multiple tissue classes to explicitly account for partial volume effects (PVE). By including isotropic response functions for non-white matter tissues such as grey matter and CSF, we can obtain a probabilistic segmentation of these tissues in the raw diffusion data, as illustrated in Fig. 6. As such, our GT method only requires a (rough) brain mask, whereas current global tractography methods typically require a white matter mask.

The global tractography method is, at the same time, a spatially-regularized fODF reconstruction method, adapted for multi-shell HARDI data and multiple tissue types. The fODF is produced as an ancillary result, based on the segment distribution, in a similar way as the track orientation distribution (TOD) (Dhollander et al., 2014). However, as global tractography matches the track density to the underlying data, the TOD becomes the fODF. The spatial regularization stems from the interaction between neighbouring segments, rather than voxels, which allows to adapt its scale to the structures of interest.

Finally, while the data energy of our global tractography method assumes Gaussian noise, the noise model in real data is Rice or noncentral $\chi$ distributed, depending on the acquisition, and may bias the reconstruction (Gudbjartsson and Patz, 1995; Aja-Fernández et al., 2014). However, by estimating the tissue response functions from the data under the same assumption, the kernels incorporate this noise bias as well, and represent not the actual tissue response, but rather expectations of the actual response under non-Gaussian noise. As such, our data-driven approach counteracts some of the effects of a Rician noise bias under the more general assumption of stationary (spatially dependent) noise. In accelerated imaging techniques, the noise distribution is not stationary, but can be well approximated as Gaussian for sufficiently high SNR (AjaFernández et al., 2014). Both datasets used in this work are of sufficient quality to support this approximation: for dataset 1 (HCP), Sotiropoulos et al. (2013) reported SNR values around 10 for shell $b=3000 \mathrm{~s} / \mathrm{mm}^{2}$; for (in-house) dataset 2, Veraart et al. (2015) estimated the median SNR 


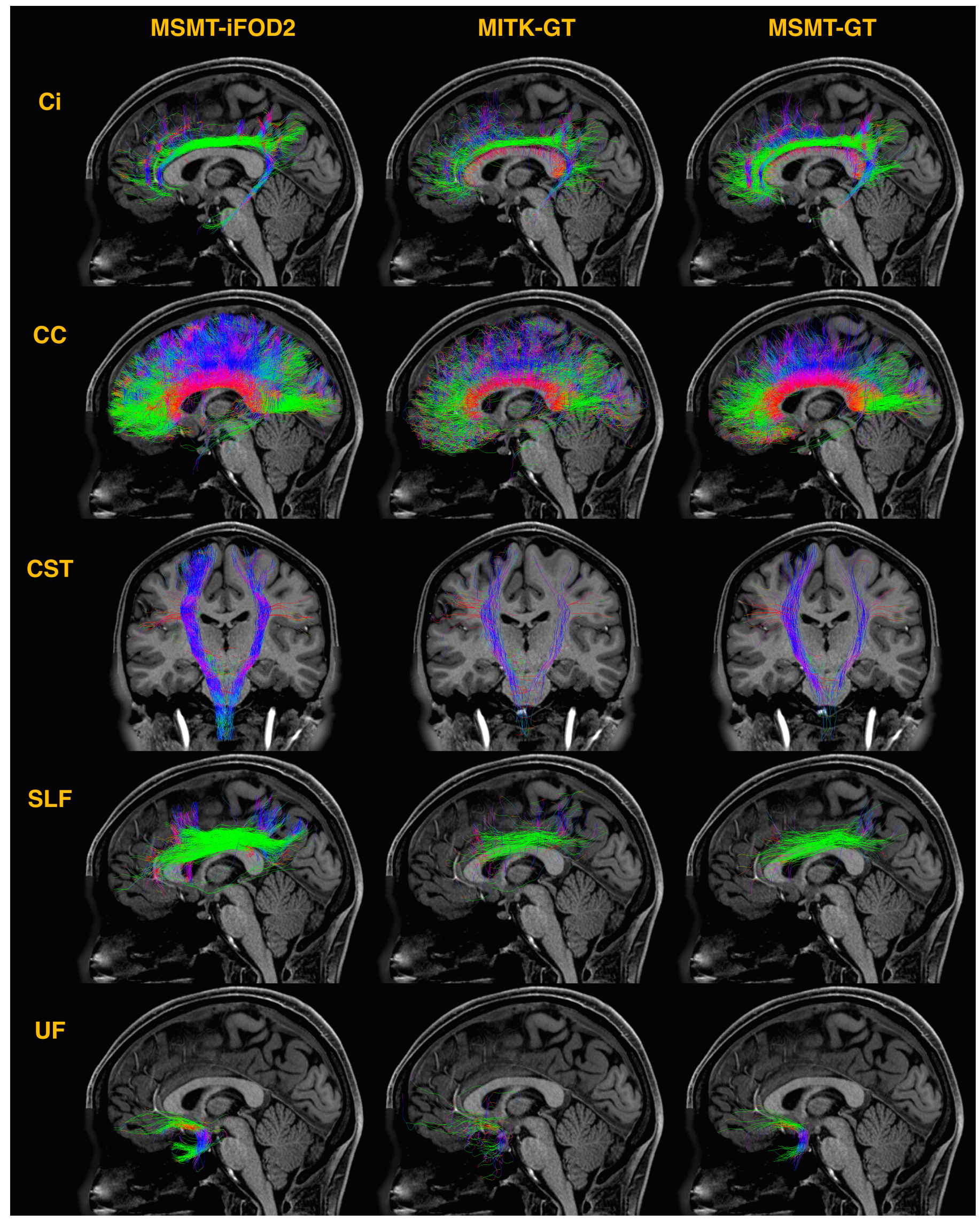

Figure 10: Segmented fibre bundles in dataset 1. From top to bottom: the right cingulate tract (Ci), the corpus callosum (CC), the left and right corticospinal tracts (CST), the right superior longitudinal fasciculus (SLF), and the right uncinate fasciculus (UF). 
at 8.42 for shell $b=2800 \mathrm{~s} / \mathrm{mm}^{2}$.

\subsection{Response function estimation}

In this work, we have used a supervised kernel estimation method, based on tissue segmentations obtained from a T1w-image. The advantage of such approach is that it allows the user to carefully select the desired kernels. The disadvantage is that T1w-data needs to be available and needs to be aligned to the DWI data ${ }^{1}$. When this is not the case, tissue classes might be segmented directly on the DWI data (Li et al., 2006; Lu et al., 2008) or a number of representative (low PVE) voxels can be manually delineated. Alternatively, one can resort to an unsupervised method, independent of any prior tissue segmentation. In Appendix A, we describe a method, based on non-negative matrix factorization, in which an arbitrary number of isotropic kernels are automatically estimated from the data. The resulting kernels closely match those of the supervised procedure, as does the global fibre reconstruction.

One limitation of the selected WM/GM/CSF representation, is that spurious segments can occur in non-WM regions that are not explicitly modelled, particularly in the dura mater and the cerebellar tentorium. Trapped water in these membranes yields a low signal attenuation with increasing $b$-value, which is most closely represented by the WM kernel. As shown in Fig. 2, white matter has the lowest attenuation of the modelled tissues, and therefore fits well in highly restrictive compartments. This touches upon a fundamental limitation of the multi-shell multi-tissue model, which we call kernel conditioning. The $b$-value attenuation of all kernels must be sufficiently distinctive to allow robust segmentation of the corresponding tissue types. Nevertheless, the effect of not modelling surrounding tissues such as the dura can be largely mediated with a proper brain mask and with the use of the particle potential.

\subsection{Tractometer results}

Tractometer analysis of the effect of the particle and connection potential has shown that no single parameter setting performs best by all measures. Instead, they can be tuned by the user depending on the application. For example, for connectome analysis one could maximize $\mathrm{VC} /(\mathrm{VC}+\mathrm{IC})$ by decreasing the connection potential, at the cost of a reduced total connection rate. For bundle segmentation, on the other hand, a higher connection potential could increase VC (to be segmented) at the cost of a higher IC (to be excluded by the segmentation). Similar observations have been made in streamline tractography, where it has been pointed out that no single parameter setting for, e.g., the step size or the maximum curvature, optimizes all tractometer metrics (Côté et al., 2013; Houde et al., 2014).

\footnotetext{
${ }^{1}$ Actually, the DWI data should be corrected for EPI distortions, for example using a reverse-phase encoding acquisition protocol (Andersson et al., 2003).
}

The comparison to alternative methods shown in Fig. 4 demonstrates that the presented approach yields a high valid connection ratio and low IB. Compared to the balland-stick model used in MITK, the multi-shell multi-tissue model strongly improves the reconstruction, especially in the low SNR range. As shown in Fig. 5, explicitly accounting for multiple tissue types in global tractography reduces spurious fibres in GM regions. Additionally, we found that the improvement in VB between MITK and our approach also relates to PVE, particularly in bundles near phantom "CSF" regions. Furthermore, calibrating the white matter model to the data at hand helps to increase $\mathrm{VC}$ and reduce $\mathrm{NC}$, which ultimately reflects in an improved $\mathrm{VC} /(\mathrm{VC}+\mathrm{IC})$ ratio.

Compared to probabilistic streamline tractography on fODFs obtained from MSMT-CSD (Jeurissen et al., 2014), our method has a higher valid connection rate but a lower total connectivity rate. This relates to a general issue with global tractography that tracks can start or end in WM due to their segment representation. Moreover, this phantom is inherently difficult for global tractography, as it assumes constant AFD in crossings (without increasing the volume of the crossing bundles), which is in conflict with the superposition of segments in our model. Streamline tracking, which does not ensure corresponding fibre density, is not affected by this assumption of the phantom.

\subsection{In vivo results}

The resulting full brain reconstructions are consistent with known anatomy, and correctly trace challenging structures, including the anterior commissure and short association fibres. The volume fraction maps shown in Fig. 6 exhibit the contrast expected from these tissues. However, the segment density contains a slight decrease in crossing regions, whereas the grey matter fraction is elevated at the corresponding positions. The WM signal in crossings can be more isotropic and can therefore more closely resemble grey matter, especially in combination with the particle potential. However, the reconstruction of the semioval centre (Fig. 7) shows that this effect is small in practice.

Figs. 7-9 illustrate that the presented method explicitly models partial volume effects between neighbouring tissues, and hence produces fODFs that are qualitatively similar to results obtained from MSMT-CSD (Jeurissen et al., 2014). However, while MSMT-CSD optimizes the data likelihood only, MSMT-GT imposes additional priors on the particles and connections. As such, additional spatial regularization and sparsity is achieved. For example, in Fig. 9, the particle potential has slightly suppressed the track reconstruction at the WM-GM interface, where the data provides less evidence, and hence avoided false connections through thin sulci. Similarly, the connection potential increases support for segments that align well with their neighbouring segments. Ultimately, these priors aim to improve the specificity of the track reconstruction.

For the purpose of bundle segmentation, the results of MSMT-GT are on par with probabilistic streamline tractog- 
raphy and show improvement over MITK-GT. Streamline tractography delineates the long-range WM bundles nicely, facilitated by the absence of any requirement to match AFD. MSMT-GT was able to reconstruct similar fibre bundles, except for the inferior part of the CST that is missing from the segmentation. By comparison, MITK-GT produced less organized tracks, particularly in the corpus callosum.

The track length histograms in Fig. 11 show a strongly skewed distribution, with high prevalence of short fibres and much less long-range connections. This result supports existing hypotheses about the efficient organisation of the brain in which proximate regions are more densely connected (Sporns, 2000; Chklovskii et al., 2002; Kaiser and Hilgetag, 2006; Raj and Chen, 2011), and is consistent with recent observations by Smith et al. (2015). Nevertheless, it is yet unclear if this truly represents the distribution of the neuronal fibre length, and to which extent it is affected by the global tractography method.

\subsection{Future perspectives}

The proposed method is calibrated to the data at hand, via a kernel that represents healthy tissue across the entire brain. A first area of future research lies in evaluating the method on pathological cases, possibly including kernels for tumour and edema tissue. Another extension would be to adapt the fibre response function to the local microstructure by allowing some degrees of freedom, in line with recent work by Reisert et al. (2014).

Secondly, global tractography may benefit from including additional priors on the expected track configuration, e.g., enforcing anatomical constraints (Smith et al., 2012; Lemkaddem et al., 2014) or imposing a prior on the local track orientation (Christiaens et al., 2014). Such priors may improve the quality of the track reconstruction or increase its robustness against low resolution or low SNR data. Furthermore, the ability of our method to simultaneously segment the WM-GM interface without relying on atlases or other imaging modalities opens new possibilities for defining priors on, e.g., track bending or endpoints.

\section{Conclusion}

The goal of this paper was to introduce a data-driven approach to global tractography, independent of a specific biophysical model. Instead, we rely on multi-shell tissue response functions, estimated from the data itself and adapted to the subject or study, for jointly reconstructing white matter fibres and the fODF, and estimating volume fractions of adjacent tissues. The findings that we have presented suggest that calibrating the fibre response and handling partial voluming in this way improves the quality of tractography. As such, we have integrated local and global reconstruction of white matter, matched to the apparent fibre density in the DWI data, and segmentation of grey matter and CSF into one comprehensive framework. The presented work is an important step towards quantitative tractography, and ultimately assessing white matter structure and connectivity in healthy subjects and patients.

\section{Appendix A. Unsupervised kernel estimation}

The estimation of the isotropic kernels for CSF and GM as described in Section 2.2 was based on on a tissue segmentation from a T1-weighted image. Here, we propose an alternative, unsupervised kernel estimation method that relies only on the DWI data at hand.

We average the raw diffusion signal on every shell to obtain, in each voxel $\vec{r}$, a vector of the mean isotropic diffusion signal per $b$-value, $\hat{D}(\vec{r}, b)$. Then, by the isotropic part of $(7), \hat{D} \approx C F$, in which $C$ is a matrix containing the isotropic kernels for all tissues, including WM, in its columns, and $F$ contains the isotropic fractions of those tissues. We then minimize $\|\hat{D}-C F\|^{2}$, s.t. $C>0, F>$ $0, \mathbf{1}^{T} F=1$, and impose the additional constraint that all kernels should be a convex combination of the original data points $\hat{D}$. This problem is commonly known as convex non-negative matrix factorization (CNMF) (Ding et al., 2010).

We took a random subsample of 3000 voxels within the brain mask of dataset 1 , and solved the CNMF problem using the iterative algorithm outlined in Ding et al. (2010). The kernels were initialized with the centroids of a k-means clustering in the 4-dimensional space of this dataset ( $b$-values). When using 4 kernels, we obtained a close correspondence to the original WM, GM and CSF kernels used in this paper, as well as a fourth kernel of low attenuation that corresponds to a highly restrictive compartment (Fig. A.12 left). The right part of Fig. A.12 shows the weights by which each voxel contributes to the kernels of corresponding colour, which converge to a sparse set in CNMF (Ding et al., 2010). The voxels contributing to the CSF and GM kernels are indeed sampled in the respective tissues. The fourth component is sampled in the dura. This illustrates that it is possible to estimate the tissue kernels from the DWI data, even when no T1w-image is available.

\section{Acknowledgements}

D. Christiaens is supported by a Ph.D. grant of the Agency for Innovation by Science and Technology (IWT). This work is financially supported by KU Leuvens Concerted Research Action GOA/11/006. Data were provided in part by the Human Connectome Project, WU-Minn Consortium (Principal Investigators: David Van Essen and Kamil Ugurbil; 1U54MH091657) funded by the 16 NIH Institutes and Centers that support the NIH Blueprint for Neuroscience Research; and by the McDonnell Center for Systems Neuroscience at Washington University. The authors also wish to thank Emmanuel Caruyer for providing the software phantom, Thibo Billiet for providing in house dataset 2, and Jelle Veraart for estimating the noise level in this dataset. 

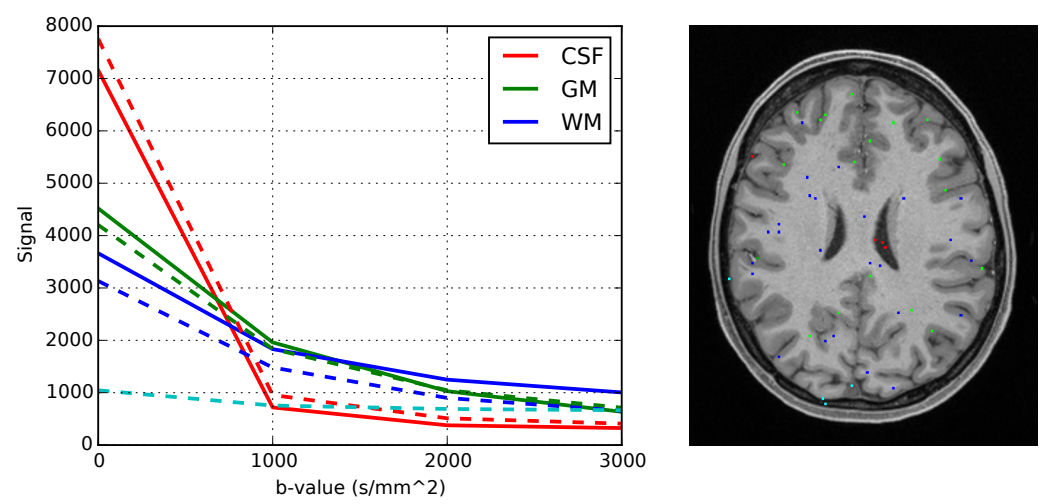

Figure A.12: Unsupervised kernel estimation. Left: Plot of the isotropic kernels estimated as described in Section 2.2 and shown in Fig. 2 (solid lines), and the four kernels estimated by the unsupervised method (dashed lines) on dataset 1 . Right: Voxels of which these kernels are a weighted average, depicted in the corresponding colours.

\section{References}

Aganj, I., Lenglet, C., Sapiro, G., Yacoub, E., Ugurbil, K., Harel, N., 2010. Reconstruction of the orientation distribution function in single- and multiple-shell q-ball imaging within constant solid angle. Magn. Reson. Med. 64, 554-566. doi:10.1002/mrm. 22365.

Aja-Fernández, S., Vegas-Sánchez-Ferrero, G., Tristán-Vega, A., 2014. Noise estimation in parallel MRI: GRAPPA and SENSE. Magnetic Resonance Imaging 32, 281-290. doi:10.1016/j.mri.2013.12.001.

Andersson, J.L., Skare, S., Ashburner, J., 2003. How to correct susceptibility distortions in spin-echo echo-planar images: application to diffusion tensor imaging. NeuroImage 20, 870-888. doi:10.1016/s1053-8119(03)00336-7.

Assaf, Y., Alexander, D.C., Jones, D.K., Bizzi, A., Behrens, T.E., Clark, C.A., Cohen, Y., Dyrby, T.B., Huppi, P.S., Knoesche, T.R., LeBihan, D., Parker, G.J., Poupon, C., 2013. The CONNECT project: Combining macro- and micro-structure. NeuroImage 80, 273-282. doi:10.1016/j.neuroimage.2013.05.055.

Basser, P., Mattiello, J., Le Bihan, D., 1994. MR diffusion tensor spectroscopy and imaging. Biophysical Journal 66, 259-267. doi:10. 1016/s0006-3495(94)80775-1.

Beaulieu, C., 2002. The basis of anisotropic water diffusion in the nervous system - a technical review. NMR in Biomedicine 15, 435-455. doi:10.1002/nbm. 782.

Behrens, T., Woolrich, M., Jenkinson, M., Johansen-Berg, H., Nunes, R., Clare, S., Matthews, P., Brady, J., Smith, S., 2003. Characterization and propagation of uncertainty in diffusion-weighted MR imaging. Magn. Reson. Med. 50, 1077-1088. doi:10.1002/mrm. 10609.

Caruyer, E., Daducci, A., Descoteaux, M., Houde, J.C., Thiran, J.P., Verma, R., 2014. Phantomas: a flexible software library to simulate diffusion MR phantoms, in: Proc. of ISMRM 2014, Milan, Italy. p. 2666. URL: http://hal.inria.fr/hal-00944644.

Chklovskii, D.B., Schikorski, T., Stevens, C.F., 2002. Wiring optimization in cortical circuits. Neuron 34, 341-347. doi:10.1016/ s0896-6273(02)00679-7.

Christiaens, D., Reisert, M., Dhollander, T., Maes, F., Sunaert, S., Suetens, P., 2014. Atlas-guided global tractography: imposing a prior on the local tract orientation, in: O'Donnell, L., Nedjati-Gilani, G., Rathi, Y., Reisert, M., Schneider, T. (Eds.), Computational Diffusion MRI. Springer International Publishing. Mathematics and Vizualisation, pp. 115-123. doi:10.1007/ 978-3-319-11182-7_11.

Côté, M.A., Girard, G., Boré, A., Garyfallidis, E., Houde, J.C., Descoteaux, M., 2013. Tractometer: Towards validation of tractography pipelines. Medical Image Analysis 17, 844-857. doi:10.1016/j.media.2013.03.009.

Daducci, A., Caruyer, E., Descoteaux, M., Houde, J.C., Thiran, J.P. (Eds.), 2013. IEEE International Symposium on Biomedical
Imaging (ISBI): HARDI Reconstruction Challenge. URL: http: //hardi.epfl.ch/static/events/2013_ISBI/.

Daducci, A., Dal Palù, A., Alia, L., Thiran, J.P., 2014. COMMIT: Convex optimization modeling for micro-structure informed tractography. IEEE Transactions on Medical Imaging , 246257doi:10.1109/tmi.2014.2352414.

Dell'Acqua, F., Catani, M., 2012. Structural human brain networks: hot topics in diffusion tractography. Current Opinion in Neurology 25, 375-383. doi:10.1097/wco.0b013e328355d544.

Dell'Acqua, F., Simmons, A., Williams, S.C., Catani, M., 2013. Can spherical deconvolution provide more information than fiber orientations? Hindrance modulated orientational anisotropy, a truetract specific index to characterize white matter diffusion. Human Brain Mapping 34, 2464-2483. doi:10.1002/hbm. 22080.

Descoteaux, M., Deriche, R., Knosche, T., Anwander, A., 2009. Deterministic and probabilistic tractography based on complex fibre orientation distributions. IEEE Transactions on Medical Imaging 28, 269-286. doi:10.1109/tmi.2008.2004424.

Dhollander, T., Emsell, L., Hecke, W.V., Maes, F., Sunaert, S., Suetens, P., 2014. Track Orientation Density Imaging (TODI) and Track Orientation Distribution (TOD) based tractography. NeuroImage 94,312-336. doi:10.1016/j.neuroimage.2013.12.047.

Ding, C., Li, T., Jordan, M., 2010. Convex and semi-nonnegative matrix factorizations. IEEE Transactions on Pattern Analysis and Machine Intelligence 32, 45-55. doi:10.1109/tpami.2008.277.

Fillard, P., Poupon, C., Mangin, J.F., 2009. A novel global tractography algorithm based on an adaptive spin glass model, in: Yang, G.Z., Hawkes, D., Rueckert, D., Noble, A., Taylor, C. (Eds.), Medical Image Computing and Computer-Assisted Intervention - MICCAI 2009. Springer Berlin Heidelberg. volume 5761 of Lecture Notes in Computer Science, pp. 927-934. doi:10.1007/978-3-642-04268-3_114.

Fritzsche, K.H., Neher, P.F., Reicht, I., van Bruggen, T., Goch, C., Reisert, M., Nolden, M., Zelzer, S., Meinzer, H.P., Stieltjes, B., 2012. MITK diffusion imaging. Methods of Information in Medicine 51, 441-448. doi:10.3414/me11-02-0031.

Girard, G., Whittingstall, K., Deriche, R., Descoteaux, M., 2014. Towards quantitative connectivity analysis: reducing tractography biases. NeuroImage 98, 266-278. doi:10.1016/j . neuroimage. 2014. 04.074 .

Glasser, M.F., Sotiropoulos, S.N., Wilson, J.A., Coalson, T.S., Fischl, B., Andersson, J.L., Xu, J., Jbabdi, S., Webster, M., Polimeni, J.R., Essen, D.C.V., Jenkinson, M., 2013. The minimal preprocessing pipelines for the Human Connectome Project. NeuroImage 80, 105-124. doi:10.1016/j.neuroimage. 2013.04.127.

Goh, A., Lenglet, C., Thompson, P.M., Vidal, R., 2009. Estimating orientation distribution functions with probability density constraints and spatial regularity, in: Yang, G.Z., Hawkes, D., Rueck- 
ert, D., Noble, A., Taylor, C. (Eds.), Medical Image Computing and Computer-Assisted Intervention - MICCAI 2009. Springer Berlin Heidelberg. volume 5761 of Lecture Notes in Computer Science, pp. 877-885. doi:10.1007/978-3-642-04268-3_108.

Green, P.J., 1995. Reversible jump Markov chain Monte Carlo computation and Bayesian model determination. Biometrika 82, 711-732. doi:10.1093/biomet/82.4.711

Gudbjartsson, H., Patz, S., 1995. The rician distribution of noisy MRI data. Magn. Reson. Med. 34, 910-914. doi:10.1002/mrm. 1910340618.

Houde, J.C., Caruyer, E., Daducci, A., Descoteaux, M., 2014. How should tractography go forward? a tractometer evaluation os local reconstruction and tracking, in: Proc. of ISMRM 2014, Milan, Italy. p. 273.

Jbabdi, S., Johansen-Berg, H., 2011. Tractography: Where do we go from here? Brain Connectivity 1, 169-183. doi:10.1089/brain. 2011.0033.

Jeurissen, B., Leemans, A., Tournier, J.D., Jones, D.K., Sijbers, J., 2012. Investigating the prevalence of complex fiber configurations in white matter tissue with diffusion magnetic resonance imaging. Human Brain Mapping 34, 2747-2766. doi:10.1002/hbm. 22099.

Jeurissen, B., Tournier, J.D., Dhollander, T., Connelly, A., Sijbers, J., 2014. Multi-tissue constrained spherical deconvolution for improved analysis of multi-shell diffusion MRI data. NeuroImage 103, 411-426. doi:10.1016/j.neuroimage. 2014.07.061.

Jones, D.K., Knösche, T.R., Turner, R., 2013. White matter integrity, fiber count, and other fallacies: The do's and don'ts of diffusion MRI. NeuroImage 73, 239-254. doi:10.1016/j .neuroimage. 2012. 06.081.

Kaiser, M., Hilgetag, C.C., 2006. Nonoptimal component placement, but short processing paths, due to long-distance projections in neural systems. PLoS Computational Biology 2, e95. doi:10.1371/ journal.pcbi.0020095.

Kreher, B.W., Mader, I., Kiselev, V.G., 2008. Gibbs tracking: A novel approach for the reconstruction of neuronal pathways. Magn. Reson. Med. 60, 953-963. doi:10.1002/mrm.21749.

Le Bihan, D., Breton, E., Lallemand, D., Grenier, P., Cabanis, E., Laval-Jeantet, M., 1986. MR imaging of intravoxel incoherent motions: application to diffusion and perfusion in neurologic disorders. Radiology 161, 401-407. doi:10.1148/radiology.161.2.3763909.

Lemkaddem, A., Skiöldebrand, D., Dal Palù, A., Thiran, J.P., Daducci, A., 2014. Global tractography with embedded anatomical priors for quantitative connectivity analysis. Frontiers in Neurology 5. doi:10.3389/fneur.2014.00232.

Li, H., Liu, T., Young, G., Guo, L., Wong, S., 2006. Brain tissue segmentation based on DWI/DTI data, in: 3rd IEEE International Symposium on Biomedical Imaging: Macro to Nano, IEEE. pp. 57-60. doi:10.1109/isbi.2006.1624851.

Lu, C.F., Wang, P.S., Chou, Y.C., Li, H.C., Soong, B.W., Wu, Y.T., 2008. Segmentation of diffusion-weighted brain images using expectation maximization algorithm initialized by hierarchical clustering, in: 2008 30th Annual International Conference of the IEEE Engineering in Medicine and Biology Society, IEEE. pp. 5502-5505. doi:10.1109/iembs. 2008.4650460.

Mangin, J.F., Fillard, P., Cointepas, Y., Bihan, D.L., Frouin, V., Poupon, C., 2013. Toward global tractography. NeuroImage 80, 290-296. doi:10.1016/j.neuroimage.2013.04.009.

Mangin, J.F., Poupon, C., Cointepas, Y., Rivière, D., PapadopoulosOrfanos, D., Clark, C.A., Régis, J., Bihan, D.L., 2002. A framework based on spin glass models for the inference of anatomical connectivity from diffusion-weighted MR data - a technical review. NMR in Biomedicine 15, 481-492. doi:10.1002/nbm.780.

Mori, S., van Zijl, P.C.M., 2002. Fiber tracking: principles and strategies - a technical review. NMR in Biomedicine 15, 468-480. doi:10.1002/nbm.781.

Neher, P.F., Stieltjes, B., Reisert, M., Reicht, I., Meinzer, H.P., Fritzsche, K.H., 2012. MITK global tractography, in: Haynor, D.R., Ourselin, S. (Eds.), Medical Imaging 2012: Image Processing, SPIE. p. 83144D. doi:10.1117/12.911215.

Panagiotaki, E., Schneider, T., Siow, B., Hall, M.G., Lythgoe, M.F., Alexander, D.C., 2012. Compartment models of the diffusion
MR signal in brain white matter: A taxonomy and comparison. NeuroImage 59, 2241-2254. doi:10.1016/j.neuroimage.2011.09. 081.

Poupon, C., Clark, C., Frouin, V., Régis, J., Bloch, I., Bihan, D.L., Mangin, J.F., 2000. Regularization of diffusion-based direction maps for the tracking of brain white matter fascicles. NeuroImage 12, 184-195. doi:10.1006/nimg. 2000.0607.

Raffelt, D., Tournier, J.D., Rose, S., Ridgway, G.R., Henderson, R., Crozier, S., Salvado, O., Connelly, A., 2012. Apparent fibre density: A novel measure for the analysis of diffusion-weighted magnetic resonance images. NeuroImage 59, 3976-3994. doi:10.1016/j. neuroimage.2011.10.045.

Raj, A., Chen, Y., 2011. The wiring economy principle: Connectivity determines anatomy in the human brain. PLoS ONE 6, e14832. doi:10.1371/journal pone. 0014832 .

Reisert, M., Kiselev, V., 2011. Fiber continuity: An anisotropic prior for ODF estimation. IEEE Transactions on Medical Imaging 30 1274-1283. doi:10.1109/tmi.2011.2112769.

Reisert, M., Kiselev, V., Dihtal, B., Kellner, E., Novikov, D., 2014. MesoFT: Unifying diffusion modelling and fiber tracking, in: Golland, P., Hata, N., Barillot, C., Hornegger, J., Howe, R. (Eds.), Medical Image Computing and Computer-Assisted Intervention - MICCAI 2014. Springer International Publishing. volume 8675 of Lecture Notes in Computer Science, pp. 201-208. doi:10.1007/978-3-319-10443-0_26.

Reisert, M., Mader, I., Anastasopoulos, C., Weigel, M., Schnell, S., Kiselev, V., 2011. Global fiber reconstruction becomes practical. NeuroImage 54, 955-962. doi:10.1016/j.neuroimage. 2010. 09.016

Sherbondy, A.J., Rowe, M.C., Alexander, D.C., 2010. MicroTrack: An algorithm for concurrent projectome and microstructure estimation, in: Jiang, T., Navab, N., Pluim, J.P.W., Viergever, M.A. (Eds.), Medical Image Computing and Computer-Assisted Intervention - MICCAI 2010. Springer Berlin Heidelberg. volume 6361 of Lecture Notes in Computer Science, pp. 183-190. doi:10.1007/978-3-642-15705-9_23.

Smith, R.E., Tournier, J.D., Calamante, F., Connelly, A., 2012. Anatomically-constrained tractography: Improved diffusion MRI streamlines tractography through effective use of anatomical information. NeuroImage 62, 1924-1938. doi:10.1016/j.neuroimage . 2012.06 .005 .

Smith, R.E., Tournier, J.D., Calamante, F., Connelly, A., 2013. SIFT: Spherical-deconvolution informed filtering of tractograms. NeuroImage 67, 298-312. doi:10.1016/j .neuroimage. 2012.11.049.

Smith, R.E., Tournier, J.D., Calamante, F., Connelly, A., 2015. The effects of SIFT on the reproducibility and biological accuracy of the structural connectome. NeuroImage 104, 253-265. doi:10.1016/j. neuroimage.2014.10.004.

Smith, S.M., Jenkinson, M., Woolrich, M.W., Beckmann, C.F., Behrens, T.E., Johansen-Berg, H., Bannister, P.R., Luca, M.D., Drobnjak, I., Flitney, D.E., Niazy, R.K., Saunders, J., Vickers, J., Zhang, Y., Stefano, N.D., Brady, J.M., Matthews, P.M., 2004. Advances in functional and structural MR image analysis and implementation as FSL. NeuroImage 23, S208-S219. doi:10.1016/j.neuroimage. 2004.07.051.

Sotiropoulos, S.N., Jbabdi, S., Xu, J., Andersson, J.L., Moeller, S., Auerbach, E.J., Glasser, M.F., Hernandez, M., Sapiro, G., Jenkinson, M., Feinberg, D.A., Yacoub, E., Lenglet, C., Essen, D.C.V., Ugurbil, K., Behrens, T.E., 2013. Advances in diffusion MRI acquisition and processing in the human connectome project. NeuroImage 80,125-143. doi:10.1016/j.neuroimage.2013.05.057.

Sporns, O., 2000. Theoretical neuroanatomy: Relating anatomical and functional connectivity in graphs and cortical connection matrices. Cerebral Cortex 10, 127-141. doi:10.1093/cercor/10.2.127.

Sporns, O., Tononi, G., Kötter, R., 2005. The human connectome: A structural description of the human brain. PLoS Computational Biology 1, e42. doi:10.1371/journal.pcbi.0010042.

Tournier, J.D., Calamante, F., Connelly, A., 2007. Robust determination of the fibre orientation distribution in diffusion MRI: Nonnegativity constrained super-resolved spherical deconvolution. NeuroImage 35, 1459-1472. doi:10.1016/j.neuroimage .2007.02.016. 
Tournier, J.D., Calamante, F., Connelly, A., 2010. Improved probabilistic streamlines tractography by 2 nd order integration over fibre orientation distributions, in: Proceedings of the International Society for Magnetic Resonance in Medicine, p. 1670.

Tournier, J.D., Calamante, F., Connelly, A., 2012. MRtrix: Diffusion tractography in crossing fiber regions. International Journal of Imaging Systems and Technology 22, 53-66. doi:10.1002/ima. 22005.

Tournier, J.D., Mori, S., Leemans, A., 2011. Diffusion tensor imaging and beyond. Magn. Reson. Med. 65, 1532-1556. doi:10.1002/mrm. 22924.

Tuch, D.S., Reese, T.G., Wiegell, M.R., Makris, N., Belliveau, J.W., Wedeen, V.J., 2002. High angular resolution diffusion imaging reveals intravoxel white matter fiber heterogeneity. Magn. Reson. Med. 48, 577-582. doi:10.1002/mrm. 10268.

Van Essen, D.C., Smith, S.M., Barch, D.M., Behrens, T.E., Yacoub, E., Ugurbil, K., 2013. The WU-Minn human connectome project: An overview. NeuroImage 80,62-79. doi:10.1016/j.neuroimage. 2013.05 .041$.

Veraart, J., Fieremans, E., Novikov, D.S., 2015. Noise map estimation in diffusion MRI using random matrix theory, in: Proc. of ISMRM 2015, Toronto, Canada. p. 1023.

Wassermann, D., Makris, N., Rathi, Y., Shenton, M., Kikinis, R., Kubicki, M., Westin, C.F., 2013. On describing human white matter anatomy: The white matter query language, in: Mori, K., Sakuma, I., Sato, Y., Barillot, C., Navab, N. (Eds.), Medical Image Computing and Computer-Assisted Intervention - MICCAI 2013. Springer Berlin Heidelberg. volume 8149 of Lecture Notes in Computer Science, pp. 647-654. doi:10.1007/978-3-642-40811-3_ 81.

Zhang, Y., Brady, M., Smith, S., 2001. Segmentation of brain MR images through a hidden markov random field model and the expectation-maximization algorithm. IEEE Transactions on Medical Imaging 20, 45-57. doi:10.1109/42.906424.

Zhou, Q., Michailovich, O.V., Rathi, Y., 2014. Spatially regularized reconstruction of fibre orientation distributions in the presence of isotropic diffusion. Computing Research Repository abs/1401.6196. URL: http://arxiv.org/abs/1401.6196. 